\title{
Advancements in polymer science: 'smart' drug delivery systems for the treatment of cancer
}

\section{Abstract}

The therapeutic potential of polymeric nanoparticles has garnered attention due to the multiple applications for which this technology can be used. This is particularly important for cancer as many of the cytotoxic drugs that are used to treat patients have negative side effects on healthy cells. Polymeric nanoparticle technology can reduce these negative side effects as they can be engineered to respond to the unique external environment surrounding tumors (i.e. an acidic environment and $\mathrm{pH}$ response) or they can target specific receptors such as folate, found exclusively on cancer cells and release their payload directly. This review will discuss the current applications of polymer nanoparticles in nanomedicine as a whole, with a focus on the development of polymeric nanoparticles and their applications as targeted drug delivery vehicles.

\section{Graphical abstract}

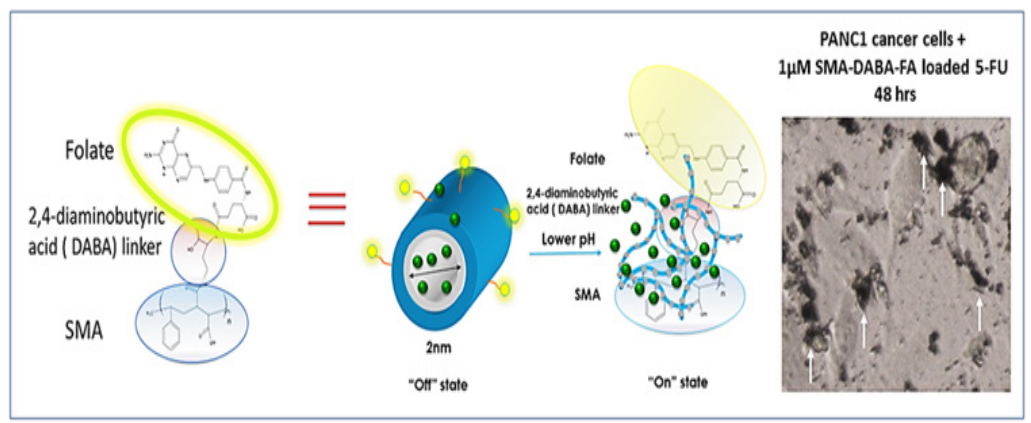

Keywords: nanoparticles, targeted drug delivery systems, $\mathrm{pH}$ sensitive delivery vehicle, slow release, nanomedicine, pancreatic cancer
Volume I Issue 3 - 2017

\author{
Manpreet Sambi, ${ }^{1,2}$ Bessi Qorri,, ${ }^{1,2}$ Cecile \\ Malardier-Jugroot, ${ }^{3}$ Myron R Szewczuk' \\ 'Department of Biomedical and Molecular Sciences, Queen's \\ University, Kingston, ON, Canada. \\ ${ }^{2}$ Contributing first authorship \\ ${ }^{3}$ Department of Chemistry and Chemical Engineering, Royal \\ Military College of Canada, Kingston, ON, Canada
}

\begin{abstract}
Correspondence: Myron R Szewczuk, Department of Biomedical and Molecular Sciences, Queen's University, Kingston, ON K7L 3N6, Canada, Tel + I 613533 2457, Fax + | 6I35336796,Email szewczuk@queensu.ca
\end{abstract}

Cecile Malardier-Jugroot, Department of Chemistry and Chemical Engineering, Sawyer Mod 5, Rm 55 I2, Royal Military College of Canada, PO Box 17000, Station Forces Kingston, ON K7K 7B4, Canada, Tel + | 613 54I 6000 ext 6046, Fax + | 613 542 9489,Email Cecile.Malardier-Jugroot@rmc.ca

Received: May 20, 2017 | Published: July II, 2017
Abbreviations: PSMA, poly(styrene-alt-maleic anhydride); FA-SMA, folate functionalized SMA; DABA, 2,4-diaminobutyric acid; PLGA-OP, oseltamivir phosphate poly(lactic-co-glycolic acid); OP-pPEGMEMA, oseltamivir phosphate-conjugated polymeric micelles

\section{Introduction}

Conventional chemotherapy is one of the standard, treatments for cancer patients; however, current strategies used in the clinic suffer from limitations such as poor aqueous solubility, elevated toxicity, lack of cancer cell selectivity and chemoresistance. ${ }^{1}$ The application of nanotechnology to deliver drugs to tumor cells is an attractive alternative. It allows drugs to be delivered to cancer cells specifically with prolonged circulation time and with a controlled drug release. ${ }^{2-4}$ In addition, controlled drug delivery improves bioavailability by preventing premature degradation and enhancing uptake, as well as maintaining drug concentration, release rates within the therapeutic window, and reducing side effects..$^{5-7}$ Another advantage of nanomaterials is the possibility to design materials with amphiphilic properties allowing for the solubilization of potent hydrophobic drugs for efficient transport and delivery. Therefore, different strategies have been adopted to develop nanocarriers specifically for the delivery of chemotherapeutic drugs. This paper will briefly introduce the use of nanomaterials in medicine, general methods used for the optimization of targeted drug carriers, and will present in detail three successful carriers developed for the delivery of chemotherapeutic drugs.

\section{Nanoparticles in medicine}

Nanoparticles are nano- or micro-sized composed of metal or organic elements that can be fabricated from synthetic, semi-synthetic or natural materials for use in several applications in the field of medicine. Firstly, metal nanoparticles, such as gold or silver, have been successfully used for imaging as well as biosensing. ${ }^{8}$ These applications rely on the novel physical and optical properties of the particles at the nanoscale. Indeed, gold nanoparticles are optically active and their absorption wavelength can be controlled with the size of the nanoparticles. This property is linked to the surface Plasmon band of the unpaired electrons at the surface of the nanoparticle, which gives a very precise and wide range of colors accessible for very efficient imaging and sensing properties. Secondly, nanoparticles have potential applications in regenerative medicine using mesoporous silica nanoparticles. ${ }^{9}$ This platform may be used for imaging in tissue engineering and for stem cell therapy as well as controlled drug release, however, the potential applications require an evaluation of the biocompatibility of the mesoporous silica nanoparticles.

\section{Nanoparticles in drug delivery}

The application of nanotechnology to deliver drugs using polymeric templates commonly utilizes three levels of targeting 
strategies: passive, active and stimuli responsive targeting. Passive targeting is known as the enhanced permeability and retention (EPR) effect, which takes advantage of the leaky vasculature of solid tumor structures. This EPR allows particles with a certain size to enter and accumulate in tumor tissues. ${ }^{7,10}$ The biocompatible polymers following unloading of the drug would eventually clear through the excretory system. Despite the ability of the EPR effect to improve the accumulation of drugs, there are several barriers to the EPR effect including high interstitial fluid pressure in tumor tissues, ${ }^{2}$ layers of tissue penetration that is demanded for therapeutic drugs, and liver and spleen accumulation of these particles. As a result, additional targeting strategies are required to enhance the distribution of the therapeutic macromolecules. Active targeting mechanisms utilize tumor-specific receptor ligands to achieve a degree of specificity and therefore it is used as a promising complementary strategy to EPR effect. Targeting molecules include carbohydrates, antibodies and ligands where their receptors are overly expressed in tumor tissues but are limited in healthy cells. Active targeting would not only improve therapeutic efficiency but also enable a reduction of the amount of drug that must be administered to achieve a therapeutic response, thus minimizing negative side effects. ${ }^{2,11}$ Finally, stimuli-responsive delivery systems are designed to respond to external stimuli (i.e., $\mathrm{pH}$, light, magnetic field) so that drug release is only triggered at the desired time and location. When exposed to external stimuli, the polymers will undergo physiochemical structural changes, and therefore lose the welldefined nanoarchitectures, releasing drugs directly into tumor cells. Among the external stimuli, $\mathrm{pH}$ gradients have been widely used for controlled release relying on the abnormally low $\mathrm{pH}$ of endosomes and tumor tissues compared to healthy tissues. ${ }^{12-15}$

To further enhance their capabilities, "smart" polymers and nanoparticles are capable of controlled release of their cargo as well as being able to target specific sites in order to efficiently deliver therapies. Polymers provide advantages over other cancer immunotherapies if they can load both tumor macromolecules and adjuvants, and through specific sizes and functional moieties be selectively delivered to specific target sites. The surfaces of these polymers are engineered to elicit immune responses facilitating antigen presentation. ${ }^{16} \mathrm{By}$ adding specific functionalities, engineered nanoparticles can present a more sensitive delivery vehicle that reduces the toxicity of its cargo by directly targeting the intended cell type, and indirectly increasing the efficacy of a drug by maintaining the integrity of healthy cells. Cancer is an area to which polymer science is currently being applied. Chemotherapy is highly toxic to both cancer and healthy cells. Therefore, in an effort to make chemotherapy more effective, nanoparticles that are designed to target specific receptors that are exclusively expressed on cancer cells are currently being developed.

\section{Design and synthesis of the drug carrier}

Although a broad range of applications are being studied for nanoparticles in medicine, ${ }^{17}$ the main development, has been focused on the synthesis of efficient targeted drug delivery carriers. The main synthetic vehicles for drug delivery are composed of polymeric nanoparticles. Polymers are easy to synthesize with specific functionalizations which can be inserted on the chain. The polymers can be biocompatible, but the main advantage of polymers in drug delivery lies in the wide number of shapes accessible by self-assembly and their biomimic properties of base-pair recognition. Indeed, polymers can self-assemble into a variety of shapes from a spherical micelle to gyroids or lamella with hydrophobic or hydrophilic cores of controllable sizes. These nanoparticles can be tailored for a specific application, particularly developed for the delivery of different drugs using oral treatment. ${ }^{18}$ This report has shown that the size and shape of nanoparticles can have a significant impact on the efficiency of treatment. The large surface area of nanoparticles presents as an advantage for efficient interactions with the gastrointestinal tract compared with common vehicles used for oral treatment.

The first polymeric carriers developed used EPR effect for preferential accumulation of the carrier and the drug close to the cancer cells due to the vasculature of the fast reproducing cells (Figure 1). The efficiency of these carriers varied broadly depending on their molecular weight and their release mechanism.

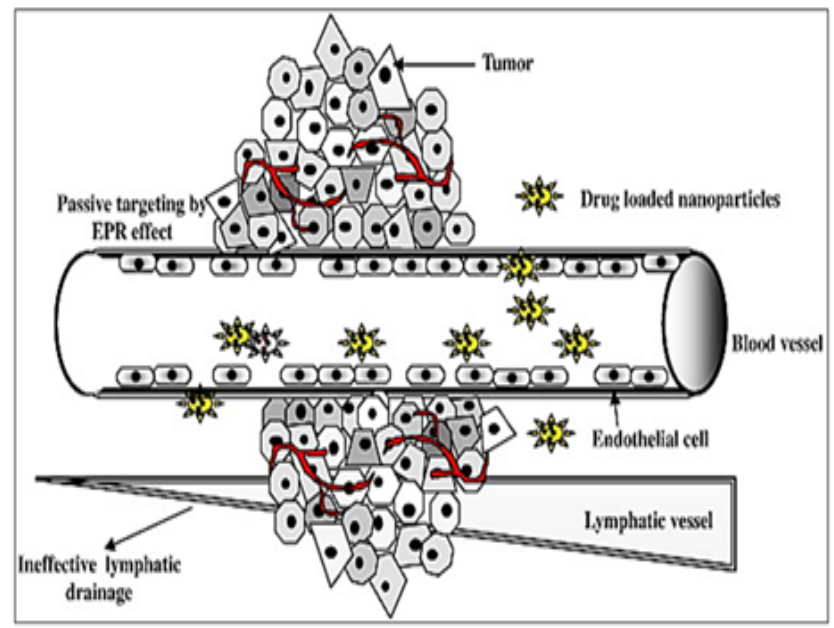

Figure I Passive targeting of nanoparticles (NP) by EPR effect utilizing the anatomical and pathophysiological abnormalities of tumor vasculature. Reproduced with permission from Acharya and Sahoo. ${ }^{24}$

A second approach for the targeting strategy was by functionalization of the polymer for a specific response to a change in their environment, including $\mathrm{pH}$, ionic strength, magnetic field, and heat. Specific functionalization of a polymer can provide specific environmentally responsive physical properties. Functionalization in general can be designed to enhance the photocatalytic properties of a material for solar cell applications,${ }^{19}$ the optical and magnetic responsiveness of nanomaterials can also be controlled through functionalization ${ }^{20}$ and functionalization of biodegradable polymers was used successfully for tissue engineering applications. ${ }^{21}$ In the field of drug delivery, the functionalization of a nanostructure can be designed for a direct release of the drug. The drug is often covalently linked to the polymer and changes in the microenvironment can trigger cleavage of the polymer-drug bond, releasing the drug. This approach requires a chemical reaction on-site and all the cleavable parts of the drug-carrier complex to be biocompatible. A more promising approach for the targeted release of hydrophobic drugs uses a functionalized carrier encapsulating the hydrophobic drug in its core that can be targeted towards specific cells.

\section{The efficacy of polymers as targeted drug de- livery systems}

\section{Response to external stimuli}

In recent years, technological advancements have been geared towards manufacturing devices that not only perform at peak efficiency, but are also capable of displaying a form of intelligence. This trend is 
currently being applied to the development of drug delivery systems in hopes of creating a polymer compound that is not only more efficient at administering chemotherapeutic drugs but also has the means to respond to external environmental stimuli. It is necessary to control and guide the interactions between these nanoparticles and the environment in which they are engineered to exert their therapeutic effects. ${ }^{22}$ This is a particularly important paradigm shift in the field of cancer research as current treatment options, such as chemotherapy, have adverse side effects on healthy cells due to systemic delivery of the chemotherapeutics. Chemotherapeutic agents are typically small hydrophobic molecules that exert their intracellular effects to inhibit an aspect of cell division. The hydrophobicity of many of these drugs results in failure in clinical trials due to poor pharmacokinetics. ${ }^{23} \mathrm{As}$ a result, the development of amphiphilic nanocarriers has allowed for a targeted delivery of these hydrophobic drugs. This delivery system can be designed with a hydrophobic interior and hydrophilic exterior, which allows it to encapsulate hydrophobic agents, such as chemotherapeutic drugs, while maintaining its own solubility in an aqueous environment. Amphiphilic polymeric vessels can provide an advantage to deliver these potent drugs to specific targets, minimizing negative side effects.

Gaps are present in the endothelial cells of tumor blood vessels. Nanoparticles take advantage of this leaky vasculature to extravagate efficiently into tumor tissues, and with the absence of lymphatic drainage from the tumors, it contributes to retention of the nanocarriers, ultimately leading to accumulation of high drug concentrations.

These loaded nanoparticles can also result in passive immunity based on drug accumulation around the tumor. However, the properties of the tumor microenvironment and vasculature have recently been exploited such that drug release from the nanoparticles occurs strictly at the tumor site. Tumors have a particularly acidic microenvironment with a $\mathrm{pH}$ of 6.5-6.9, which is uncharacteristic of normal, healthy tissues ( $\mathrm{pH} 7.2-7.4) .{ }^{25}$ To this end, a $\mathrm{pH}$ responsive amphiphilic nanocarrier was developed that is capable of modifying its structural integrity upon exposure to a change in $\mathrm{pH}^{5,6}$ The unique characteristic of poly(styrene-alt-maleic anhydride) (PSMA) is such that at a neutral $\mathrm{pH}$, the polymer forms stable nanoparticles which are capable of encapsulating hydrophobic agents in the core and are able to release their cargo by undergoing a conformational change upon exposure to a change in $\mathrm{pH}$. This allows for a more specific delivery of potent therapeutics. In addition, the stable structure obtained by self-assembly at $\mathrm{pH} 7$ can be tuned for specific drugs with either a tubular hydrophobic core with an inner diameter of 3 $\mathrm{nm}$ or a hydrophobic core delimited by two hydrophobic lamella at a distance of $3 \mathrm{~nm} .{ }^{26}$ The self-assembly of alternating copolymers presents significant advantages compared to block copolymers which were the main component of the first polymeric nanocarriers. Indeed, the self-assembly of alternating copolymers do not require the addition of a co-solvent such as dimethyl sulfoxide (DMSO) and thus, no critical micelle concentrations have been reported for selfassembly. These properties ensure a simple one-step process for the encapsulation of the hydrophobic drug as well as a complete control of the concentration of both the polymer and drug.

Another unique characteristic of nanomedicine is that it can be engineered for active targeting such that they can interact with target cells via specific ligand-receptor interactions. The folate receptor is highly expressed on many cancer cell types such as the kidney, lung, breast, bladder and pancreas. ${ }^{27}$ Due to its elevated expression in malignant cell types, it presents as a valuable therapeutic target in nanomedicine and allows the carrier to preferentially bind to cancerous cells prior to endocytosis. Recently, the poly(styrene-altmaleic anhydride) (PMSA) amphiphilic polymer has been further modified and functionalized with the addition of folic acid, generating a folic acid functionalized amphiphilic alternating copolymer (FADABA-SMA). As with the PSMA molecule, this functionalized polymer not only maintains its $\mathrm{pH}$ responsiveness which promotes drug release after endocytosis, but is also able to bind to the overexpressed folate receptors present on cancer cells. ${ }^{28}$ This property allows for this delivery system to take advantage of the characteristics of the human body such that the intact cylinder can travel through the body, and release the drugs following internalization by malignant cells where the $\mathrm{pH}$ changes. ${ }^{29}$ Following folate binding to its receptor, the nanoparticle is internalized in an endocytic pathway into the cytosol, where the $\mathrm{pH}$ drops further to $\mathrm{pH} 5 .{ }^{30}$ Due to the amphiphilic nature of this conjugated nanoparticle, this $\mathrm{pH}$ drop will result in a conformational change that will release the therapeutic load directly into the malignant cell (Figure 2). This specific release has two main advantages: the potent hydrophobic drug transported directly into the cancerous cell will not affect the healthy cells with a significant reduction of the side effects related to chemotherapy, and will allow for a decrease in the drug concentration needed for the desired effect on the cancerous cells.

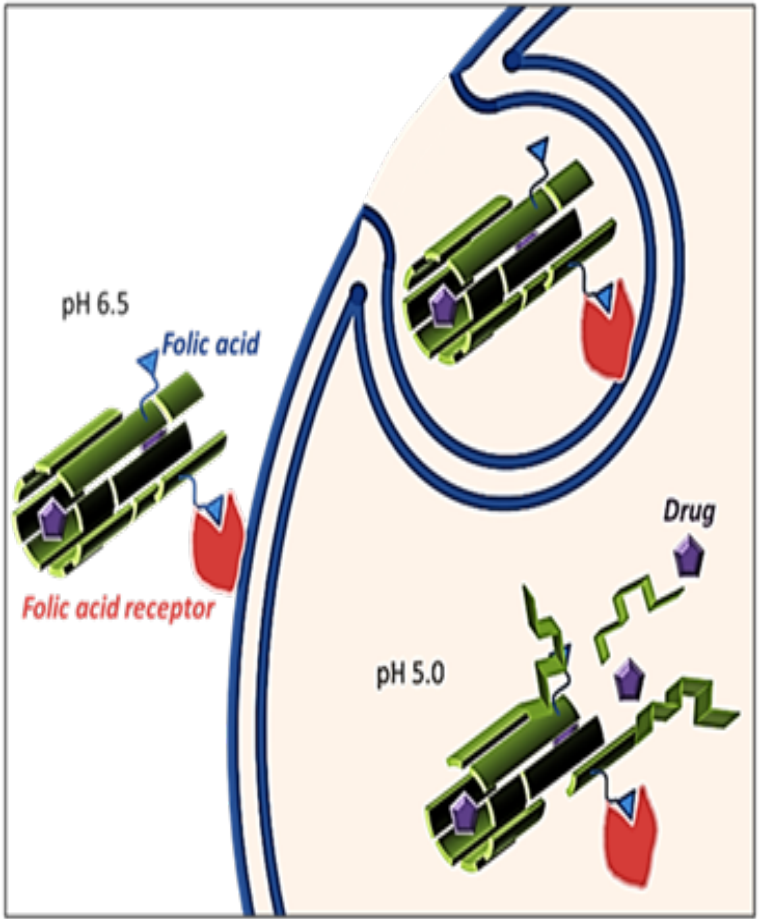

Figure 2 Schematic representation of targeted delivery through folic acidmediated receptor endocytosis of the nanocarrier and release of the drug due to conformational changes of the polymer in a low $\mathrm{pH}$ environment.

To test the efficacy of the FA-DABA-SMA delivery system, when administered in vitro to RAW-Blue macrophage and PANC1 pancreatic cancer cell lines, curcumin loaded SMA was shown to aggregate and surround the cells for uptake into the cytoplasm. However, the curcumin was not found to be present in the nucleus. In contrast, FA-DABA-SMA was internalized through folic acidmediated receptor endocytosis and curcumin was found to have been 
internalized and localized to the nucleus through fluorescence analysis (Figure 3). ${ }^{29}$ In addition, the study showed no adverse effects of the functionalized polymer on cell viability. However, the cell viability study of the curcumin loaded FA-DABA-SMA revealed a significant decrease in the number of cancerous cells. Clinically, these findings have relevant implications as many chemotherapeutic drugs are cytotoxic to cells because they target and inhibit different oncogenic processes. Therefore, one possible avenue to further explore would be to encapsulate hydrophobic chemotherapeutic drugs and determine whether there is an increase in cell death when compared with conventional methods of treatment.

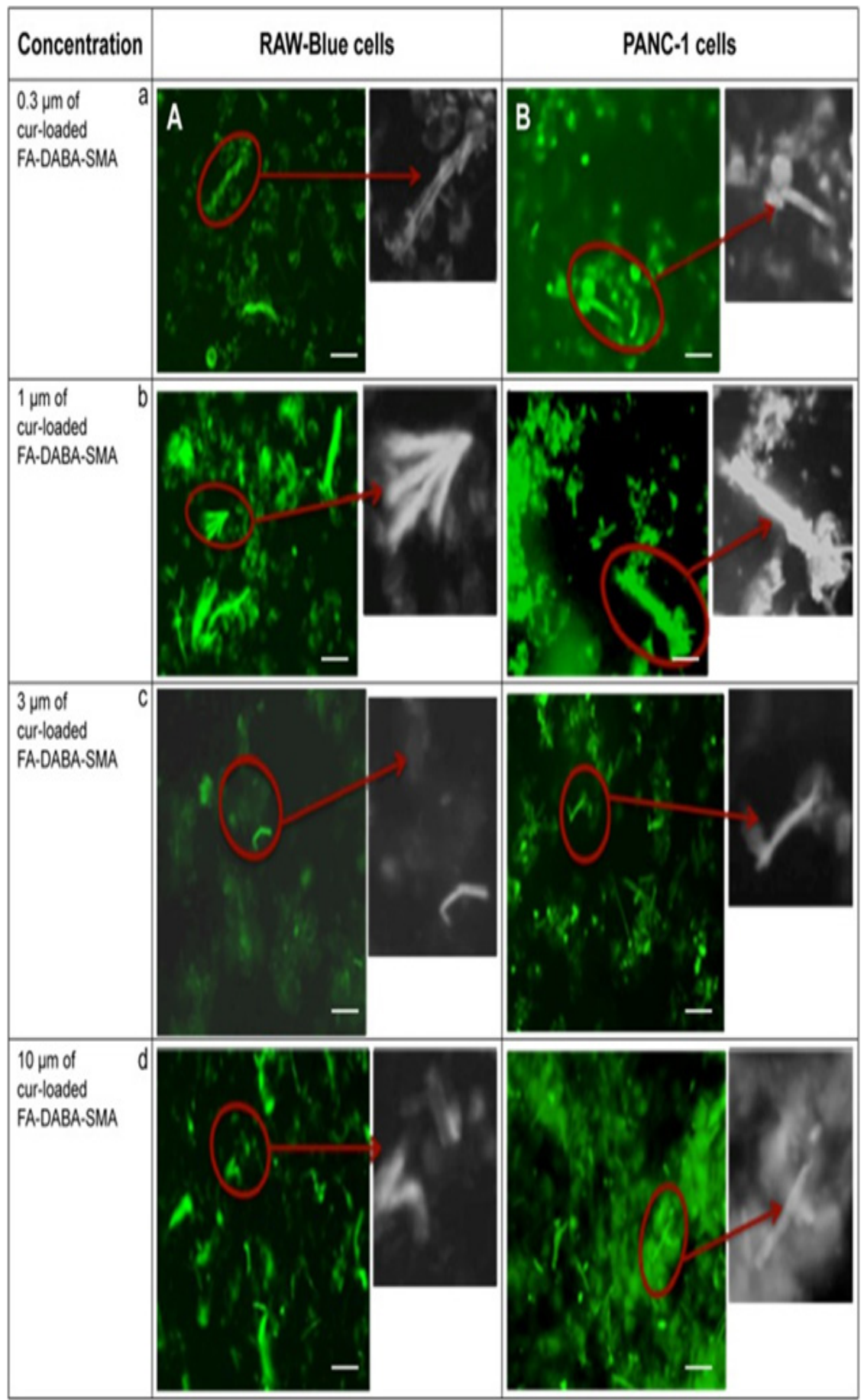

Figure 3 Fluorescent images showing cellular uptake of curcumin-loaded FA-SMA in (A) RAW-Blue and (B) PANC-I cell lines. Notes: Images in the left column indicate

(A) RAW-Blue cell line with concentrations of (a) 0.3 , (b) I, (c) 3 , and (d) $10 \mu \mathrm{m}$ of curcumin-loaded FA-SMA, respectively, and images in the right column indicate (B) PANC-I cell line with concentrations of (a) 0.3 , (b) I, (c) 3 , and (d) $10 \mu \mathrm{m}$ of curcumin-loaded FA-SMA, respectively. The image bar scale represents I00 $\mu \mathrm{m}$. Taken in part from: $@$ Li et al..$^{29}$ Publisher and licensee Dove Medical Press Ltd. This is an Open Access article which permits unrestricted non-commercial use, provided the original work is properly cited. 
The targeted drug delivery system developed and optimized in this study shows very good potential for efficient release of hydrophobic chemotherapeutic agents directly to the cancerous cell without affecting normal cells and minimizing the negative side effects. However, this drug carrier could also be used for imaging and early detection of cancerous cells. Indeed, the template formed by the unfunctionalized polymer promotes the synthesis of optically active gold nanoclusters $(2-3 \mathrm{~nm}$ in diameter) as well as monoatomically thin gold nanosheets $(\sim 50 \mathrm{~nm}$ in diameter $){ }^{26,31}$ The combination of a targeted carrier (FA-DABA-SMA) and an optically active material (gold decorated SMA) is expected to improve the sensitivity of cancer cell imaging.

\section{Slow release drug delivery}

In addition to poor pharmacokinetics due to the hydrophobic nature of many cancer therapies, cancer treatment involves multiple doses of these drug cocktails as intravenous (IV) injections. As a result, nanoscale polymeric drug delivery systems such as polymeric micelles and liposomes have been developed for targeted and sustained release of chemotherapeutic drugs. The development of poly (lacticco-glycolide) (PLGA) nanoparticles allows for the slow release of therapeutic agents over a course of days up to weeks. ${ }^{32}$ PLGA is synthesized through a copolymerization reaction that involves the ring opening of two different monomers of glycolic and lactic acid. ${ }^{32}$ During the polymerization process, monomers are linked through ester bonds and contribute to PLGA's biodegradability because this linkage undergoes hydrolysis in the body and generates products that are normally produced through normal metabolic processes. ${ }^{32}$

Recently, we have designed and developed a PLGA cylinder loaded with oseltamivir phosphate (PLGA-OP).$^{33}$ Based on reported studies, we have provided evidence of the crucial role of epidermal growth factor receptor (EGFR) in initiating a number of processes involved in cancer progression including angiogenesis, metastasis, chemoresistance and tumorigenesis. ${ }^{34}$ Furthermore, we have shown the significant efficacy of OP in inhibiting neuraminidase-1 (Neu-1) in order to prevent the activation of these tumor-promoting signaling pathways. ${ }^{35}$ The release kinetics of PLGA-OP in biphasic release profiles via bulk erosion was shown in RAGxCy double mutant mouse models with human pancreatic PANC-1 cancer cells. OP released from PLGA-OP $20 \mathrm{mg}$ cylinders inhibited tumor growth for approximately 30 days, beyond this, tumor growth was minimal. ${ }^{33}$ In addition, very few to no metastatic clusters were seen in the liver and lung tissues of cohorts with the PLGA-OP transplant compared to the untreated control cohorts due to an inhibition of tumor neovascularization. Loss of E-cadherin is known to drive epithelial-to-mesenchymal transition. Reduced metastatic burden can be attributed to maintained E-cadherin levels and decreased N-cadherin lvels (but $\mathrm{N}$ cadherin doesn't drive EMT). This study confirmed the therapeutic potential of a PLGAdesigned cylinder delivery vehicle loaded with OP in order to reduce metastatic burden and tumor growth when implanted at the tumor site without the need for repeated drug administration.

\section{Polymeric micelles: receptor targeting}

Polymeric micelles are a class of nanoparticles that are able to encapsulate hydrophobic drugs in their core due to their amphiphilic nature and are generally smaller than $100 \mathrm{~nm}$ in size. ${ }^{36}$ The assembly of these structures follows a similar pattern seen in viruses and lipoproteins. Their therapeutic potential was recognized in 1984 when they were first proposed as vehicles to deliver antitumor drugs in a safe and minimally invasive manner, particularly because of the high demand for low density lipoproteins by tumors. ${ }^{37}$ Furthermore, these micelles can be conjugated with compounds that can target specific receptors on tumor cells, thereby making these nanoparticles particularly useful in targeted drug delivery applications.

Our group has developed OP-conjugated polymeric micelles (OP-pPEGMEMA) which demonstrated a concentration-dependent inhibition of sialidase activity, using a microbial $(\alpha-2,3)$ neuraminidase from Streptococcus pneumoniae. ${ }^{38}$ To expand these targeted effects on the free enzyme, PANC-1 pancreatic cancer cells treated with soluble OP-pPEGMEMA conjugates in a dose-dependent manner achieved up to $45 \%$ reduction in cell viability. The OP-conjugated polymeric micelles specifically targeted Neu- 1 similar to that of free OP. ${ }^{38}$ These OP-conjugated micelles targeting Neu-1 in complex with EGFRs provided a novel way of specific targeting and delivering chemotherapeutic agents by receptor-induced internalization of OPmicelles via Neu-1 binding. ${ }^{39}$

\section{Conclusion}

Targeted drug delivery systems hold promise in efficiently targeting tumors. This is particularly important for tumors that are unresectable. Due to their unique design and capacity to encapsulate drugs and release them slowly over time or at a specific site of action, polymeric nanoparticles are an exciting field of study that is currently being applied to cancer therapies. In addition, clinical conventional approved anticancer drugs can be loaded and tested in these polymers to study their solubility, cellular uptake, and cytotoxicity. With increasing modifications being applied to these "smart" polymeric particles to make their targeting more efficient and specific in their response to external stimuli, polymer science is continually evolving in order to provide an efficacious mode for the treatment of cancer. The most promising drug carriers are those that combine both active and passive targeting strategies. The strategy presented in this review with a folic acid functionalized, $\mathrm{pH}$-sensitive polymer highlights the specificity and efficiency of drug release with a decrease in the number of cancerous cells with curcumin used as the drug. The functionalization of the polymeric carrier has the potential to be adapted for specific receptors and may include different targeting centers for a highly responsive and "smart" strategy towards efficient cancer treatment.

\section{Acknowledgements}

None.

\section{Conflict of interest}

The author declares no conflict of interest.

\section{References}

1. Chidambaram M, Manavalan R, Kathiresan K. Nanotherapeutics to overcome conventional cancer chemotherapy limitations. J Pharm Pharm Sci. 2011;14(1):67-77.

2. Bertrand $\mathrm{N}, \mathrm{Wu} \mathrm{J}, \mathrm{Xu} \mathrm{X}$, et al. Cancer nanotechnology: the impact of passive and active targeting in the era of modern cancer biology. Adv Drug Deliv Rev. 2014;66:2-25.

3. Brannon Peppas L, Blanchette JO. Nanoparticle and targeted systems for cancer therapy. Adv Drug Deliv Rev. 2004;56(11):1649-1659. 
4. Bae YH, Park K. Targeted drug delivery to tumors: myths, reality and possibility. J Control Release. 2011;153(3):198-205.

5. Malardier Jugroot C, Van De Ven TGM, Whitehead MA. Novel self-assembly of amphiphilic copolymers into nanotubes: characterization by small-angle neutron scattering. Langmuir. 2005;21(22):10179-10187.

6. Malardier Jugroot C, Van De Ven TG, Whitehead MA. Linear conformation of poly (styrene-alt-maleic anhydride) capable of self-assembly: a result of chain stiffening by internal hydrogen bonds. J Phys Chem B. 2005;109(15):7022-7032.

7. Maeda H, Nakamura H, Fang J. The EPR effect for macromolecular drug delivery to solid tumors: Improvement of tumor uptake, lowering of systemic toxicity, and distinct tumor imaging in vivo. Adv Drug Deliv Rev. 2013;65(1):71-79.

8. Kairdolf BA, Qian X, Nie S. Bioconjugated nanoparticles for biosensing, in vivo imaging, and medical diagnostics. Anal Chem. 2017;89(2):1015-1031.

9. Rosenholm JM, Zhang J, Linden M, et al. Mesoporous silica nanoparticles in tissue engineering--a perspective. Nanomedicine (Lond) 2016;11(4):391-402.

10. Zhang Y, Chan HF, Leong KW. Advanced materials and processing for drug delivery: the past and the future. Advanced drug delivery reviews. 2013;65(1):104-120.

11. Allen TM, Cullis PR. Drug delivery systems: entering the mainstream. Science. 2004;303(5665):1818-1822.

12. Maeda H. SMANCS and polymer-conjugated macromolecular drugs: advantages in cancer chemotherapy. Adv Drug Deliv Rev. 2001;46(13):169-185.

13. Brown SD, Nativo P, Smith JA, et al. Gold nanoparticles for the improved anticancer drug delivery of the active component of oxaliplatin. $J$ Am Chem. 2010;132(13):4678-4684.

14. Rejinold NS, Thomas RG, Muthiah M, et al. Radio frequency triggered curcumin delivery from thermo and $\mathrm{pH}$ responsive nanoparticles containing gold nanoparticles and its in vivo localization studies in an orthotopic breast tumor model. RSC Advances. 2014;4(74):39408-39427.

15. Gao W, Chan JM, Farokhzad OC. pH-Responsive nanoparticles for drug delivery. Molecular pharmaceutics. 2010;7(6):1913-1920.

16. Fan L, Campagnoli S, Wu H, et al. Negatively charged AuNP modified with monoclonal antibody against novel tumor antigen FAT1 for tumor targeting. J Exp Clin Cancer Res. 2015;34:103.

17. Reddy KR, Gomes VG. Nanoparticles for Clinical Applications. Vol 2015 11th ed. USA: American Scientific Publishers; 2015.

18. Banerjee A, Berezhkovskii A, Nossal R. Kinetics of cellular uptake of viruses and nanoparticles via clathrin-mediated endocytosis. Phys Biol. 2016;13(1):016005.

19. Reddy KR, Hassan M, Gomes VG. Hybrid nanostructures based on titanium dioxide for enhanced photocatalysis. Applied Catalysis A: General. 2015;489:1-16.

20. Reddy KR, Lee K-P, Gopalan AI, et al. Facile synthesis of hollow spheres of sulfonated polyanilines. Polym J. 2006;38(4):349-354.

21. Macková $H$, Plichta Z, Hlídková $H$, et al. Reductively degradable poly(2-hydroxyethyl methacrylate) hydrogels with oriented porosity for tissue engineering applications. ACS Applied Materials \& Interfaces. 2017;9(12):10544-10553.

22. Mout R, Moyano DF, Rana S, et al. Surface functionalization of nanoparticles for nanomedicine. Chem Soc Rev. 2012;41(7):2539-2544.
23. Savic R, Eisenberg A, Maysinger D. Block copolymer micelles as delivery vehicles of hydrophobic drugs: micelle-cell interactions. J Drug Target. 2006;14(6):343-355.

24. Acharya S, Sahoo SK. PLGA nanoparticles containing various anticancer agents and tumour delivery by EPR effect. Advanced Drug Delivery Reviews. 2011;63(3):170-183

25. Estrella V, Chen T, Lloyd M, et al. Acidity generated by the tumor microenvironment drives local invasion. Cancer Res. 2013;73(5):1524 1535 .

26. McTaggart M, Malardier-Jugroot C, Jugroot M. Self-assembled biomimetic nanoreactors I: Polymeric template. Chemical Physics Letters. 2015;636:216-220.

27. Parker N, Turk MJ, Westrick E, et al. Folate receptor expression in carcinomas and normal tissues determined by a quantitative radioligand binding assay. Anal Biochem. 2005;338(2):284-293.

28. Lee JH, Jung HW, Kang IK, et al. Cell behaviour on polymer surfaces with different functional groups. Biomaterials. 1994;15(9):705-711.

29. Li X, Szewczuk MR, Malardier Jugroot C. Folic acid-conjugated amphiphilic alternating copolymer as a new active tumor targeting drug delivery platform. Drug Des Devel Ther. 2016;10:4101-4110.

30. Mansoori GA, Brandenburg KS, Shakeri Zadeh A. A comparative study of two folate-conjugated gold nanoparticles for cancer nanotechnology applications. Cancers (Basel). 2010;2(4):1911-1928.

31. McTaggart M, Malardier Jugroot C, Jugroot M. Self-assembled biomimetic nanoreactors II: Noble metal active centers. Chemical Physics Letters. 2015;636:221-227.

32. Lu JM, Wang X, Marin-Muller C, et al. Current advances in research and clinical applications of PLGA-based nanotechnology. Expert review of molecular diagnostics. 2009;9(4):325-341.

33. Hrynyk M, Ellis JP, Haxho F, et al. Therapeutic designed poly (lactic-co-glycolic acid) cylindrical oseltamivir phosphate-loaded implants impede tumor neovascularization, growth and metastasis in mouse model of human pancreatic carcinoma. Drug design, development and therapy. 2015;9:4573-4586.

34. Gilmour AM, Abdulkhalek S, Cheng TS, et al. A novel epidermal growth factor receptor-signaling platform and its targeted translation in pancreatic cancer. Cell Signal. 2013;25(12):2587-2603.

35. Abdulkhalek S, Olivia Geen, Lacey Brodhagen, et al. Transcriptional factor Snail controls tumor neovascularization, growth and metastasis in mouse model of human ovarian carcinoma. Clinical and Translational Medicine. 2014;3(1):28.

36. Jones N, Dumont DJ. Recruitment of Dok-R to the EGF receptor through its PTB domain is required for attenuation of Erk MAP kinase activation. Curr Biol. 1999;9(18):1057-1060.

37. Samadi Baboli M, Favre G, Canal P, et al. Low density lipoprotein for cytotoxic drug targeting: improved activity of elliptinium derivative against B16 melanoma in mice. Br J Cancer. 1993;68(2):319-326.

38. Kapishon V, Allison S, Whitney RA, et al. Oseltamivir-conjugated polymeric micelles prepared by RAFT living radical polymerization as a new active tumor targeting drug delivery platform. Biomater Sci. 2016;4(3):511-521.

39. Haxho F, Neufeld RJ, Szewczuk MR. Neuraminidase-1: A novel therapeutic target in multistage tumorigenesis. Oncotarget. 2016;7(26):40860-40881. 